\title{
Improving Psychosexual Knowledge in Adolescents with Autism Spectrum Disorder: Pilot of the Tackling Teenage Training Program
}

\author{
Linda P. Dekker • Esther J. M. van der Vegt • Kirsten Visser • \\ Nouchka Tick • Frieda Boudesteijn · Frank C. Verhulst • \\ Athanasios Maras $\cdot$ Kirstin Greaves-Lord
}

Published online: 16 November 2014

(C) Springer Science+Business Media New York 2014

\begin{abstract}
Previous studies have shown that psychosexual functioning in adolescents with autism spectrum disorder (ASD) is hampered and emphasize the need for a specialized training program tailored to their needs. Therefore, an individual training program was developed; the Tackling Teenage Training (TTT) program. The current pilot study systematically evaluated whether psychosexual knowledge increased after taking part in the TTT program, using a preand post-training design in 30 adolescents with ASD (77 \% male, mean age $=14.80$ years, mean intelligence $=96.96$ ). Psychosexual knowledge increased significantly (pre-training total score: $M=25.74, S D=6.20$; post-training total score: $M=33.52 \quad(S D=2.78)$; $\mathrm{F}(1,29)=65.20, p<.001)$. The TTT program may be useful to improve psychosexual knowledge and functioning in adolescents with ASD, yet these findings are preliminary, and a more elaborate controlled trial is needed.
\end{abstract}

Keywords Psychosexual functioning - Autism spectrum disorder $\cdot$ Psychosexual knowledge $\cdot$ Training program

Electronic supplementary material The online version of this article (doi:10.1007/s10803-014-2301-9) contains supplementary material, which is available to authorized users.

L. P. Dekker $(凹) \cdot$ E. J. M. van der Vegt · K. Visser · N. Tick ·

F. C. Verhulst - K. Greaves-Lord

Department of Child and Adolescent Psychiatry/Psychology,

Erasmus MC-Sophia, Wytemaweg 8, Room KP 2881,

3015 CN Rotterdam, The Netherlands

e-mail: 1.p.dekker@erasmusmc.nl

L. P. Dekker - E. J. M. van der Vegt - K. Visser - N. Tick ·

F. Boudesteijn - A. Maras - K. Greaves-Lord

Yulius Academy and Yulius Autism, Yulius, Mental Health

Organisation, Mathenesserlaan 202, 3013 HH Rotterdam,

The Netherlands

\section{Introduction}

Due to the main characteristics of autism spectrum disorder (ASD), namely impairments in social interaction and communication as well as restricted, repetitive and stereotyped behaviors (APA 2013), individuals with ASD run into a variety of difficulties in daily life. The discrepancy between their physical development and their socio-emotional development, may increasingly lead to difficulties as individuals with ASD mature (Kuo et al. 2011; Anderson et al. 2011; Sullivan and Caterino 2008), especially with regard to psychosexual functioning (Gougeon 2010). Psychosexual functioning is a multi-facet concept covering not merely sexual behaviors (i.e. behaviors and experiences with sexuality), but also sexual selfhood (i.e. the internal functioning of people, including for example sexual knowledge and self-esteem) and sexual socialization (i.e. the context in which psychosexual development takes place, such as friends, family and the internet; Dewinter et al. 2013; Tolman and McClelland 2011). Many professionals as well as parents report problems and worries in relation to psychosexual functioning of adolescents with ASD, such as limited awareness of personal and public boundaries (e.g. Ballan 2012; Hellemans et al. 2007; Stokes et al. 2007). The problems in psychosexual functioning may originate from limited knowledge and skills with respect to appropriate psychosexual functioning (Stokes et al. 2007; Sullivan and Caterino 2008; Stokes and Kaur 2005).

A putative reason for the difficulty in psychosexual functioning, has been suggested to be the result of the implicit rules in particular contexts that often accompany sexual situations (Hénault 2006). As implicit learning of social cues is weakened in adolescents with ASD (Hudson et al. 2012), these adolescents usually need explicit 
information to acquire the appropriate knowledge and skills, also regarding sexual functioning (Gougeon 2010).

Previous studies suggest that guidance and education on psychosexual functioning for adolescents with ASD may help in stimulating healthy psychosexual functioning (Gougeon 2010; Hénault 2006). The need to develop an appropriate training program on psychosexual functioning, that is tailored to the needs of individuals with ASD, has been emphasized by several authors (e.g. Gougeon 2010; Hellemans et al. 2007). However, to our knowledge, to date, no evidence-based individual training program on psychosexual functioning exists specifically tailored to the needs and difficulties of adolescents with ASD which offers guidance with tackling the particular difficulties that adolescents with ASD may run into.

For this purpose, we developed an individual training program; the Tackling Teenage Training (TTT) program (Boudesteijn et al. 2011). This training program consists of 18 individual sessions in which a variety of psychosexual topics are discussed (i.e. psycho-education) alternated with exercises (e.g. behavioral rehearsal; see "Methods" section for full description) and is specifically tailored to the needs of cognitively able adolescents with ASD. The TTT program aims to improve all domains of psychosexual functioning (i.e. sexual behavior, sexual selfhood, and sexual socialization), with a particular focus on obtaining theoretical as well as practical knowledge (e.g. explicit explanation of often implicit rules and practicing conversational skills).

Healthy psychosexual functioning requires specific knowledge and skills in all domains (i.e. sexual behavior, sexual selfhood, and sexual socialization); for example regarding personal hygiene, developing and maintaining relationships, recognizing and acknowledging personal boundaries (both of self and others), and intimacy and sexual activity with or without partners (Hart-Kerkhoffs et al. 2009; Hénault 2006; Maniglio 2012). Research has shown that sex education programs which positively impact sexual behavior in typically developing individuals incorporate increasing sexual knowledge (Kirby et al. 2007; Ryan et al. 2007; Rock et al. 2005). Psychosexual knowledge may therefore be important both with regard to making informed decisions, i.e. for example regarding reproduction, intimate behaviors and understanding boundaries (e.g. Urbano et al. 2013) as well as to promote healthy psychosexual behavior, such as the use of a condom (e.g. Rock et al. 2005). Therefore a specialized training program such as the TTT program (Boudesteijn et al. 2011) may be a valuable first step in increasing psychosexual knowledge to improve psychosexual functioning in adolescents with ASD.

Several studies have shown that adolescents with ASD have less knowledge regarding sexuality than typically developing (TD) peers (e.g. Hellemans et al. 2007; Stokes and Kaur 2005). Although some studies have found average psychosexual knowledge levels in individuals with ASD whom are cognitively able (Byers et al. 2013), such differences may be explained to the diverse operationalization of psychosexual knowledge, ranging from simple theoretical vocabulary knowledge (e.g. what is a 'wet dream') to broader psychosexual knowledge, such as knowledge on developing relationships (see Dewinter et al. 2013 for a comprehensive review). A recent study by Brown-Lavoie et al. (2014) using a measure on theoretical sexual knowledge (e.g. reproductive health, sexually transmitted infections, and condom use) found that individuals with ASD had significantly less perceived and less actual knowledge than controls without ASD. In addition, even with adequate theoretical knowledge, adolescents do not always transfer this knowledge into daily functioning (Hellemans et al. 2007). Potentially not only theoretical (e.g. definitions of sexual terminology) but also broader practical psychosexual knowledge and insights (e.g. how to appropriately deal with a psychosexual situations, such as personal hygiene or communication about sexual topics) is important in exhibiting the appropriate skills and psychosexual functioning. Limited knowledge on psychosexual topics in adolescents with ASD may, in certain cases, escalate into inappropriate behaviors; due to for instance limited understanding of private versus public sexual behavior (Nichols and Blakeley-Smith 2009) or even dangerous situations; for instance due to the limited ability to identify abusive behavior (Sevlever et al. 2013).

Therefore, the aim of the current pilot study was to investigate (a) whether psychosexual knowledge significantly increased after taking part in the TTT program by means of a pre-training and post-training design. Subsequently, we explored (b) which aspects of psychosexual knowledge particularly seemed to increase and (c) whether particular characteristics of the participants were related to improved psychosexual knowledge. The final aim was to (d) explore whether parents perceived that their child applied the acquired knowledge in everyday life (i.e. transfer of knowledge to adaptation of behavior).

\section{Methods}

Participants and Procedure

Participants were 30 11-19 year old adolescents with ASD $(M=14.80$ years, $S D=2.07 ; 77 \%$ male $)$. Data were collected between April 2010 and May 2012. The adolescents were in treatment at Yulius; a large expert mental health care organization providing care in the South-West of the Netherlands, which is, among other expertise, specialized in children, adolescents and (young) adults with 
Table 1 Sample characteristics

\begin{tabular}{|c|c|c|}
\hline \multirow{2}{*}{ Gender (male) } & & \multirow{2}{*}{$\frac{N(\%)}{23(77)}$} \\
\hline & & \\
\hline \multicolumn{2}{|l|}{ PPD-NOS classification ${ }^{a}$} & $24(80)$ \\
\hline \multicolumn{2}{|c|}{ Asperger's syndrome (AS) classification ${ }^{a}$} & $3(10)$ \\
\hline \multicolumn{2}{|c|}{ Autistic disorder (AD) classification $^{a}$} & $3(10)$ \\
\hline \multicolumn{2}{|c|}{$\begin{array}{l}\text { Perceived transfer of knowledge to adaptation } \\
\text { of behaviour (parent-reported 'yes') }\end{array}$} & $19(86)^{b}$ \\
\hline & $M(S D)$ & Range \\
\hline Age & $14.80(2.08)$ & $11-19$ \\
\hline Total IQ & $96.96(10.80)$ & $79-120$ \\
\hline Calibrated severity score ADOS $^{\mathrm{c}}$ & $5.46(2.92)$ & $1-10$ \\
\hline Motivation average $^{\mathrm{d}}$ & $7.33(1.15)$ & $4.88-9.67$ \\
\hline Difficulty average $^{\mathrm{d}}$ & $4.37(1.89)$ & $1.12-8.00$ \\
\hline Resistance average $^{\mathrm{d}}$ & $1.51(1.16)$ & $0-4$ \\
\hline
\end{tabular}

PDD-NOS Pervasive developmental disorder-Not otherwise specified, ADOS autism diagnostic observation schedule. Total sample is 30 adolescents

a Based on axis 1 classification of the Diagnostic and Statistical Manual of Mental Disorders, Fourth Edition, Text Revision (DSM-IV-TR; APA, 2013)

b Available for $N=21$ (70\% of total sample).

c Available for $N=22$ (73\% of total sample); Calibrated scores as described in Hus and Lord (2014); Module 4.

d Available for $N=28$ (93\% of total sample); Trainer reported about adolescent; $0=$ not at all to $10=$ very much

ASD. Participants were in treatment either at the inpatient or outpatient clinic, and were referred to the TTT program by their clinical practitioner. Referrals were made either because problems in psychosexual functioning had already occurred (i.e. intervention purposes) or because the clinician foresaw future issues regarding the psychosexual functioning of the adolescent (i.e. prevention purposes). All participants gave informed consent. The pilot study was approved by a Dutch mental health ethics committee (METIGG; NL36421.097.11).

As the TTT program was developed for cognitively able adolescents with ASD, the inclusion criteria were; a total IQ score of 75 or higher, age between 11 and 19 years old and a clinical diagnosis of ASD. Based on their Diagnostic and Statistical Manual of Mental Disorders, Fourth Edition, Text Revision (DSM-IV-TR) axis 1 classification, adolescents were classified as ASD if they fulfilled the criteria for a classification of Pervasive Developmental Disorder-Not Otherwise Specified (PDD-NOS), Asperger's syndrome (AS) or Autistic disorder (AD). The inclusion criteria were set as the training program was specifically tailored to the needs of cognitively able adolescents with ASD.

A group of 40 adolescents was eligible to start the training program and to participate in the systematic evaluation. Of 30 adolescents, we had complete data. The incomplete data of the remaining ten adolescents was due to two reasons. First, during the course of the training program, seven adolescents (17.5\%) dropped out and did not finish the training program due to personal circumstances or practical reasons (e.g. moving to a different location or scheduling of appointments was not feasible). In addition, three adolescents $(5 \%)$ did finish the training program, but did not complete the psychosexual knowledge test at both time points. The group without complete data did not differ significantly from the group with complete data on their knowledge test score at $\mathrm{T} 1[t(38)=1.87$, $p=.07], \quad$ age $[t(38)=-.73, \quad p=.47], \quad$ intelligence $[t(31)=.93, p=.36]$, or gender $\left[\chi^{2}(1, \mathrm{~N}=38)=.45\right.$, Fisher's exact test $p=.66]$. For a detailed description of our sample we refer to Table 1, which shows the sample characteristics.

To investigate whether the TTT program increased psychosexual knowledge in our sample, we used a pre-test, post-test design. We obtained data by means of a psychosexual knowledge test (described in Materials) at pretraining and post-training. On average, the training program is completed in approximately 6 months (i.e. 18 weekly sessions), although this may vary. Pre-training data were collected during an intake session 1 week before the TTT program. Post-training data were collected during an evaluative session, 1 week after finishing the final session of the TTT program.

Prior to the intake session (i.e. pre-training data collection) and the start of the training program, the adolescents and parents were invited for an introduction meeting. During the introduction meeting, the purpose of the systematic evaluation, the time-investment, the aspects of the systematic evaluation as well as the content of the training program were discussed.

At the intake session, the adolescent filled out the psychosexual knowledge test. The adolescents were able to ask questions for clarification, but did not receive feedback on his/her answers on the psychosexual knowledge test, to avoid a learning effect which could influence the measurement at the post-training time-point. A week after the last session of the TTT program, at the evaluative session, the adolescent filled out the post-training psychosexual knowledge test. The parents filled out an evaluation form in which they indicated if they perceived their child to be able to apply the knowledge, acquired in the training program, in everyday life.

From the start of the TTT program, the adolescent generally had a weekly individual session of $1 \mathrm{hr}$, in which one topic of the 18 sessions training program was covered (see "Tackling Teenage Training Program" section for more detailed information). The training program was provided by trained and certified trainers of the TTT 
program, of which there were five at the time of the systematic evaluation. The certified trainers received a 2 day train-the-trainer course and participated in interdisciplinary meetings every 3 months. Each adolescent was assigned to one trainer for the duration of the entire TTT program. After each session the trainer rated motivation, resistance and difficulty of the session for the adolescent. In our sample pre-training and post-training measurements were on average 7 months apart $(M=6.8, S D=1.76$; range 3-11 months).

\section{The Tackling Teenage Training Program}

The TTT program was designed to offer guidance and support with tackling the difficulties that adolescents with ASD may encounter regarding psychosexual functioning, by increasing their knowledge and skills on this topic (Boudesteijn et al. 2011). The TTT program is an individual training program, consisting of 18 sessions covering the following topics; discussing puberty (i.e. how and with whom, session 1), appearances (session 2), first impressions (session 3), physical and emotional developments in puberty (session 4, 5 and 6), how to become friends and maintain a friendship (session 8), falling in love and dating (session 9, 11 and 18), sexuality and sex (e.g. sexual orientation, masturbation, and (safe) intercourse; sessions 7 , $10,12,13$ ), pregnancy (session 14), setting and respecting boundaries (sessions 15 and 16) and internet use (session 17) are all discussed and practiced in a structured manner with the adolescent in one-on-one training sessions (Boudesteijn et al. 2011).

All sessions are structured in the following way: the adolescent receives information regarding different subtopics of the session (i.e. psycho-education), alternated with exercises (e.g. behavioral rehearsals, and knowledge and insight quizzes). The sessions, provide the adolescent with a leaflet with the information and exercises of the session, supported with clear life-like illustrations as well as a training kit which includes materials such as a model penis (designed for educational purposes) and condoms. This so that the theoretical knowledge can be supported with practical demonstrations and explicit skills training using concrete materials. At the end of each session, the adolescent receives a take-home assignment in which the topic of that session can be discussed/practiced outside of the context of the training program, e.g. a small interview with one of the parents on the topic of the session, or arranging a get-together with a friend. After each session, parents are informed through e-mail about the topic of the session (i.e. psycho-education) and the take-home assignment of the child. In addition, particularities (i.e. strengths and difficulties of the child) of the specific session are communicated to parents. This feedback to the parents is provided with the permission of the adolescents and is intended to enhance generalization, by preparing the parents for any questions or remarks. Both the take-home assignments and the communication with the parents are intended to foster an environment in which the adolescent can feel comfortable to discuss or practice appropriate skills (i.e. conversational) regarding psychosexual topics to enhance generalization. The TTT program manual (Boudesteijn et al. 2011) is available upon request in Dutch, English, Greek and Spanish.

Materials

\section{Psychosexual Knowledge Test}

To assess psychosexual knowledge, and possible lack thereof, we used a psychosexual knowledge test of 37 items. This test is an adapted version of an existing Dutch high school biology test regarding sexual knowledge for adolescents between the ages of 13-15 years old, who are attending pre-vocational education (in Dutch 'VMBO'; Hendriks and Meijs 2005). The questions of the psychosexual knowledge test vary from understanding the meaning of sexual terminology (e.g. What does 'orgasm' mean?), to knowledge on functionality of sex organs (e.g. What happens when someone ejaculates?), to safety related topics in sexuality (e.g. Which statement regarding condom use is correct?), to more applied psychosexual knowledge topics (e.g. Why is it important to make eye contact during social interactions?; see appendix A for the full psychosexual knowledge test, in ESM). The psychosexual knowledge test consists of 35 multiple choice questions and two openended questions in which four male reproduction body-parts and six female reproduction body-parts have to be named correctly. Each question is rated as being either correct or incorrect, which leads to a score of one point per question. The total score on the psychosexual knowledge test represents the total number of correct answers, ranging from 0 to 37. Thus the higher the total score, the more correct psychosexual knowledge the adolescent has. The internal consistency of the measure at both T1 and T2 was good (Cronbach's $\alpha \mathrm{T} 1=.87$; Cronbach's $\alpha \mathrm{T} 2=.71$ ).

\section{Individual Characteristics}

Age, gender, intelligence, ASD diagnosis and severity, level of perceived motivation for the training program and difficulty and resistance to the training program, and parental level of perceived transfer of knowledge to reallife behavior were assessed to provide a detailed description of our sample.

By means of the autism diagnostic observation schedule (ADOS; Lord et al. 2000) the ASD severity was assessed 
using the calibrated severity scores (Hus and Lord 2014). The ADOS was administered by examiners who had completed the research-training and had achieved sufficient reliability for administration and coding. All the adolescents received module 4 of the ADOS based on their age as well as language abilities.

Information on intelligence (i.e. total intelligence quotient, TIQ) was gathered by means of several standardized assessment tools, generally the Wechsler intelligence scale for children (WISC; Wechsler 2004).

As potentially the level of motivation for the training program, perceived difficulty with the sessions or resistance to a training program of an adolescent can influence learning (e.g. Wolters 2003) we considered these factors in the evaluation of the training program. Trainers rated motivation, resistance and difficulty of the adolescent after each session. First, motivation of the adolescent was scored on a scale from 0 to $10(0=$ no motivation at all; $10=$ very motivated). Second, the trainer rated how much resistance the adolescent showed during the session, again on a scale from 0 to $10(0=$ no resistance at all; $10=$ very much resistance). Last, the trainer rated how difficult that particular session was for the adolescent on a scale from 0 to 10 $(0=$ very easy to; $10=$ extremely difficult $)$. These scores for each session were subsequently summed and then averaged to compute an index of overall motivation, resistance and difficulty. During the training program, neither the parent nor the adolescent were informed about the scores.

Lastly, we explored the perceived transfer of knowledge to adaptation of behavior in a parent evaluation at the end of the TTT program. Parents were asked whether their child applied the acquired knowledge in everyday life, scoring this item either yes $(=1)$ or no $(=0)$.

\section{Analyses}

Firstly, we performed descriptive analyses in order to provide information about the diagnostic characteristics of the participants as well as on how the TTT program was generally perceived (i.e. mean scores of the trainer reported perceived motivation, difficulty and resistance) as well as how parents scored the transfer of knowledge to behavior.

Secondly, we calculated frequencies to investigate the percentage of correct answers per question of the psychosexual knowledge test, both pre-training and post-training.

Thirdly, to investigate whether psychosexual knowledge significantly increased from pre-training to post-training, we performed a repeated-measures ANOVA with the total score on the psychosexual knowledge test as the dependent variable.
Table 2 Results psychosexual knowledge test per question

\begin{tabular}{|c|c|c|}
\hline Question & $\begin{array}{l}\text { Pre- } \\
\text { training } \\
\% \text { correct }\end{array}$ & $\begin{array}{l}\text { Post- } \\
\text { training } \\
\% \text { correct }\end{array}$ \\
\hline 1. Definition of 'puberty' & 93.3 & 100.0 \\
\hline 2. Definition of 'charisma' & 80.0 & 100.0 \\
\hline $\begin{array}{l}\text { 3. Definition of 'physical trait related to } \\
\text { appearance' }\end{array}$ & 70.0 & 90.0 \\
\hline 4. Relation of dress code to sexual desire & 70.0 & 76.7 \\
\hline 5. Purpose of making eye contact & 96.7 & 100.0 \\
\hline $\begin{array}{l}\text { 6. Correct word for the primary female } \\
\text { sexual organ }\end{array}$ & 90.0 & 100.0 \\
\hline $\begin{array}{l}\text { 7. Correct word for the primary male } \\
\text { sexual organ }\end{array}$ & 100.0 & 100.0 \\
\hline $\begin{array}{l}\text { 8. Naming the elements of the female } \\
\text { reproductive area }\end{array}$ & 29.6 & 50.0 \\
\hline $\begin{array}{l}\text { 9. Naming the elements of the male } \\
\text { reproductive area }\end{array}$ & 46.4 & 66.7 \\
\hline 10. Definition of 'menstruation' & 66.7 & 83.3 \\
\hline 11. Definition of 'an erection' & 73.3 & 100.0 \\
\hline 12. Timing of an erection & 66.7 & 100.0 \\
\hline 13. Definition of 'ejaculation' & 83.3 & 93.3 \\
\hline 14. Definition of 'wet dream' & 86.7 & 96.7 \\
\hline 15. Definition of 'masturbating' & 66.7 & 90.0 \\
\hline 16. Synonym 'orgasm' & 66.7 & 100.0 \\
\hline 17. Elements of friendship & 93.3 & 100.0 \\
\hline 18. Applicability of term 'friendship' & 86.7 & 96.7 \\
\hline 19. Definition of 'attraction' & 66.7 & 80.0 \\
\hline 20. Elements of 'being in love' & 90.0 & 96.7 \\
\hline 21. Definition of 'sexual preference' & 23.3 & 80.0 \\
\hline 22. Discovering sexual preference & 50.0 & 96.7 \\
\hline 23. Definition of 'heterosexual' & 63.3 & 90.0 \\
\hline 24. Definition of 'coming out' & 53.3 & 80.0 \\
\hline 25. Description of 'flirting' & 66.7 & 96.7 \\
\hline 26. Timing of first sexual intercourse & 53.3 & 83.3 \\
\hline 27. Definition of 'the pill' & 86.7 & 100.0 \\
\hline 28. Usage of condom & 93.3 & 96.7 \\
\hline 29. Definition of STI's & 50.0 & 83.3 \\
\hline 30. Definition of 'being horny' & 73.3 & 100.0 \\
\hline 31. Utility of 'foreplay' & 40.0 & 93.3 \\
\hline 32. Definition of 'hymen' & 33.3 & 70.0 \\
\hline 33. Definition of 'impregnation' & 90.0 & 100.0 \\
\hline $\begin{array}{l}\text { 34. Knowledge about unimpregnated } \\
\text { oocyte }\end{array}$ & 53.3 & 79.3 \\
\hline $\begin{array}{l}\text { 35. Knowledge about unwanted } \\
\text { pregnancy }\end{array}$ & 86.7 & 93.3 \\
\hline $\begin{array}{l}\text { 36. Definition of 'boundary crossing } \\
\text { behaviour' }\end{array}$ & 76.7 & 100.0 \\
\hline 37. Portrayal of sex in the media & 70.0 & 96.7 \\
\hline
\end{tabular}

$\%$ reflects how many of the adolescents answered the question correctly. See appendix A for full psychosexual knowledge test (in ESM) 
Finally, to explore whether individual characteristics (i.e. age, gender, intelligence, ASD severity, motivation, resistance, difficulty, and perceived transfer of knowledge) were related to a change in psychosexual knowledge, we computed Pearson correlations between these variables and the change in total score from pre-training to post-training (i.e. post- minus pre-training score) on the psychosexual knowledge test. Due to the small sample size in our pilot, we did not have sufficient power to include the individual characteristic, which may influence the effect of the training program, in the main analyses.

\section{Results}

\section{Sample Characteristics}

Table 1 shows the characteristics of our sample as well as how the TTT program was perceived, both reported by the trainer (i.e. perceived motivation, difficulty and resistance) and by parents (i.e. if the child applied the acquired psychosexual knowledge in daily life).

\section{Changes in Psychosexual Knowledge}

Table 2 shows the proportion of adolescents with ASD who correctly answered a question, at pre-training and post-training. Pre-training, adolescents had the least psychosexual knowledge regarding sexual selfhood (i.e. sexual preference, naming the separate body parts of the female reproductive area and the function of the hymen). The proportion of correct answers on almost all questions increased, except on one question. The one question was answered correctly by all the adolescents on both pretraining and post-training (i.e. correct word for the primary male sexual organ; question 7). Psychosexual knowledge increased especially on three questions, indicated by an increase of at least $40 \%$ of the adolescents answering the questions correctly: two questions regarding sexual selfhood [i.e. the definition of sexual preference (question 21; increase of $56.7 \%$ ) and how one discovers his/her sexual preference (question 22; increase of $46.7 \%$ )] and one question regarding sexual behavior [i.e. practical knowledge on 'foreplay' (question 31; increase of $53.3 \%$ )]. On more than one-third (i.e. 13 out of $37=35 \%$ ) of the questions, after the training program all the adolescents knew the correct answer, while this proportion was 1 out of $37(=3 \%)$ before the training program.

The repeated measures ANOVA we performed on the total scores of the pre-training and post-training scores on the psychosexual knowledge test showed a significant increase in overall psychosexual knowledge from pre-
Table 3 Correlation matrix change score psychosexual knowledge test

\begin{tabular}{llr}
\hline Measure & Correlation & $p$ \\
\hline Age & -.55 & $<.01$ \\
Gender (male) & -.17 & .37 \\
Total IQ & -.30 & .17 \\
Calibrated severity score ADOS $^{\mathrm{a}}$ & -.05 & .83 \\
Motivation $^{\mathrm{b}}$ & -.03 & .89 \\
Difficulty $^{\mathrm{b}}$ & .37 & .05 \\
Resistance $^{\mathrm{b}}$ & .09 & .66 \\
Child manages to apply in their daily life, the & .13 & .59 \\
psychosexual knowledge that they acquired $^{\text {during the Tackling Teenage Training (parent- }}$ & & \\
report yes) & & \\
\hline
\end{tabular}

Pearson correlations with the change score of the psychosexual knowledge test (post-training minus pre-training score)

a Available for $N=22$ (73\% of total sample); Calibrated scores as described in Hus and Lord (2014); Module 4

b Available for $N=28$ (93\% of total sample); Trainer reported about adolescent; $0=$ not at all to $10=$ very much

c Available for $N=21$ (70\% of total sample)

training $(M=25.80, \quad S D=6.30), \quad$ to $\quad$ post-training $(M=33.80, S D=2.72 ; \mathrm{F}(1,29)=65.20, p<.001)$.

Individual Characteristics Related to the Changes in Psychosexual Knowledge

The correlations in Table 3 show that age and perceived difficulty significantly correlated with the change in total score on the psychosexual knowledge test from pre-training to post-training. Younger adolescents showed a larger increase in psychosexual knowledge $(\mathrm{r}=-.55, p<.01)$, and adolescents that seemed to have more difficulty with the content of the sessions as reported by the trainer showed a larger increase in psychosexual knowledge $(\mathrm{r}=.37, p=.05)$.

\section{Discussion}

As the research literature on psychosexual functioning in ASD is steadily increasing, evidence accumulates that adolescents with ASD have specific needs for a specialized training program regarding the area of psychosexual functioning (Gougeon 2010; Hénault 2006; Hellemans et al. 2007). However, to our knowledge, there is not yet an evidence-based individual psychosexual training program available serving these particular needs. For this purpose, we developed the TTT program (Boudesteijn et al. 2011) which is a training program regarding psychosexual functioning (i.e. sexual behavior, sexual selfhood and sexual 
socialization) that is specifically tailored to the needs and the difficulties that cognitively able adolescents with ASD have and which offers them guidance and support in their psychosexual development. As knowledge is a predictor of healthy psychosexual behavior in typically developing individuals (Ryan et al. 2007), the main aim of the current systematic evaluation was to investigate whether knowledge regarding psychosexual functioning increased after participation in the TTT program, using a pre- post-training design. In our sample of 30 11-19 year old adolescents with ASD, overall psychosexual knowledge significantly increased from pre-training to post-training. In addition we explored if particular aspects of psychosexual knowledge increased. Particularly, psychosexual knowledge regarding sexual selfhood (i.e. sexual preference) and sexual behavior (i.e. practical knowledge regarding foreplay) increased, as each had at least $40 \%$ of the adolescents improve their knowledge on these items. When exploring which characteristics influenced the increase in knowledge, it was found that younger adolescents showed a larger increase in psychosexual knowledge, as well as the adolescents that were reported by their trainer to have more difficulty. Moreover when exploring if parents perceived a transfer of knowledge to adaptation of behavior, most parents reported to perceive a transfer of the learned psychosexual knowledge to every-day life.

Appropriate psychosexual knowledge may lead to healthier decision making regarding psychosexual behavior, thereby decreasing the risk adolescents may run. Although, to our knowledge, no study has investigated if psychosexual knowledge improves decision making regarding psychosexual behavior in adolescents with ASD, there are studies in typically developing adolescents which supports this (Ryan et al. 2007; Zimet et al. 1992; Reitman et al. 1996). Psychosexual knowledge of adolescents with ASD significantly increased after taking part in the TTT program, and may therefore improve the decisions that adolescents with ASD make regarding their psychosexual functioning. This hypothesis is speculatively supported by our finding that most parents reported that they perceived transfer of knowledge to adaptation of behavior in everyday life.

Age and trainer-reported difficulty significantly correlated with the change in total score on the psychosexual knowledge test. There was a strong negative association between age and the change in total score on the psychosexual knowledge test. Thus, the younger the adolescent, the more psychosexual knowledge was obtained over the course of the TTT program. There was a moderate positive relationship with trainer reported average level of difficulty and the change in total score on the psychosexual knowledge test. Thus the more difficulty the trainer estimated the adolescent had with the total training program, the larger the increase in total score on the psychosexual knowledge test. This may mean that the training program might have the most optimal outcome with respect to knowledge in relatively young teenagers, potentially as they may have been less exposed to psychosexual information or did not learn as much from regular psychosexual information sources and therefore have a lower score at the start allowing more room to learn. In addition those that seem to have greater difficulties with the total training program according to their trainer, also show the most increase in psychosexual knowledge. Potentially this may be because those with the most difficulty have less knowledge at the start of the training program or they may have misinterpreted psychosexual information leading to incorrect knowledge and therefore need to acquire more knowledge or to improve previously learned incorrect knowledge. However in our pilot study, the lack of power does not allow for the more extensive analyses required to properly analyze this. Therefore more research is needed to corroborate these preliminary conclusions. A randomized controlled trial would be better suited to investigate if indeed the TTT program improves psychosexual function on top of the effects of regular learning opportunities in daily life (e.g. sexual education in schools, interaction with peers, parents, and siblings, psychosexual experiences, and information from online sources). In addition, a more extensive assessment battery could be useful to investigate which factors are related to the effectiveness of the training program, as well as if and how the transferal of knowledge to adaptation of behavior and skills in daily life takes place.

Due to the difficulties in social communication and implicit learning of adolescents with ASD as well as their limited amount of peer relations (Orsmond et al. 2004), a formal training program, may be best suited to improve their psychosexual functioning. In typically developing adolescents, most psychosexual knowledge is acquired from informal social sources, e.g. parents and peers (Stokes et al. 2007; Brown-Lavoie et al. 2014). However, adolescents with ASD may be less able-or less frequent in situations-to learn from informal social sources as compared to their typically developing peers (Sullivan and Caterino 2008; Stokes et al. 2007; Brown-Lavoie et al. 2014). Non-social sources, such as the media, which individuals with ASD use significantly more than those without ASD (Brown-Lavoie et al. 2014), may provide incorrect or over-romanticized information which could lead to more inappropriate behavior (Collins et al. 2004). Therefore a formal training program to improve psychosexual functioning such as the TTT program, may be particularly beneficial for psychosexual functioning in adolescents with ASD. In this training program, informal social sources (e.g. parents) are also included, since parents have unique teaching opportunities within the daily lives of the 
adolescents. To stimulate such 'co-teaching' with the parents, after each session the trainer of the TTT program provides updates to the parents with information on the topic that was covered in the session, the homework that was assigned and strengths and difficulties that their child displayed with the topic.

The TTT program is aimed at $12-18$ year old boys and girls with ASD and a total IQ of 80 or higher. However, due to differences in gender, symptom severity and intelligence within this group of adolescents with ASD, it can be valuable to customize the training program to the individual characteristics and needs of an adolescent with ASD. Therefore, trainers of the TTT program were provided with a 2 day train-the-trainer in which they were taught how to provide the training program in a standardized way, with particular suggestions on how it can be customized to the individual needs of the adolescent if necessary. As part of the standardized protocol for research it is imperative that all the sessions are covered, but given the cognitive ability and personal behaviors, experiences, contexts and particular interests of the adolescent, the order of the sessions can be altered, supplementary text and/or exercises can be included, or more time can be used to discuss a particular topic. Currently, given the personal intimate matters that the training program covers it is provided individually, but an adapted version is being created in order to provide the training program in the classroom setting so that peers and teachers can be involved in the learning process.

The current exploratory systematic evaluation has some limitations, which require caution with interpretation of the results. Firstly, the design was a non-controlled pre-training and post-training design incorporated in a care-as-usual setting, thus lacked a control group that did not undergo the training program to compare outcomes. Secondly, the sample size was relatively small and showed a large variety in diagnostic characteristics. Thirdly, our sample included very few girls. Therefore we cannot generalize our findings to females with ASD. Finally, the psychosexual knowledge test was used in exactly the same version at pre-training versus post-training assessment. The increase in knowledge may be the result of a more general learning-effect or potentially some adolescents sought out information after the pre-training assessment. Therefore our results cannot be assigned definitely to the TTT program. The current pilot study prepares and informs a more elaborate randomized controlled trial, that allows us to investigate the efficacy of the TTT program using a randomized controlled trial in a larger sample including more elaborate measures, both parent- and self-report, on psychosexual functioning (i.e. sexual behavior, sexual selfhood and sexual socialization) in everyday functioning.

Although these limitations warrant caution when interpreting these preliminary results, findings seem to indicate an increase in psychosexual knowledge after participation in the TTT program. The training program had a high coherence, and those who did drop out, did so because of circumstantial reasons. As psychosexual knowledge has been related to sexual behaviors in the general population (e.g. Somers and Paulson 2000; Ryan et al. 2007) and both knowledge and skills have been suggested to affect more general psychosexual functioning (Hart-Kerkhoffs et al. 2009; Hénault 2006; Maniglio 2012), increasing the psychosexual knowledge of adolescents with ASD seems to be a valuable first step in preparing them to develop as sexually healthy adults. The long-term effects as well as the investigation if the training program is mostly beneficial for deviant behavior or victimization should be investigated in future research. Thus, the TTT program may be a potential tool in assisting the healthy psychosexual functioning of adolescents with ASD, although further research is needed to replicate and elaborate the current findings.

Acknowledgments This project was financially supported by the Sophia Children's Hospital Fund (Grant Number 617, titled Tackling Teenage: a multicenter study on psychosexual development and intimacy in adolescents with Autism Spectrum Disorder). The professionalization of the Tackling Teenage Training was financially supported by the Anthonia Wilhelimina Fund which is a part of the Psychological Health fund (i.e. Fonds Psychische Gezondheid). We are grateful to all adolescents, their parents and the trainers who participated in this research and to everyone who worked on this project and made it possible.

\section{References}

Anderson, D. K., Maye, M. P., \& Lord, C. (2011). Changes in maladaptive behaviors from midchildhood to young adulthood in autism spectrum disorder. American Journal on Intellectual and Developmental Disabilities, 116(5), 381-397.

APA. (2013). Diagnostic and statistical manual of mental disorders (DSM-5) (5th ed.). Arlington, VA: American Psychiatric Publishing.

Ballan, M. S. (2012). Parental perspectives of communication about sexuality in families of children with autism spectrum disorders. Journal of Autism and Developmental Disorders, 42(5), 676-684.

Boudesteijn, F., Van der Vegt, E. J. M., Visser, K., Tick, N., \& Maras, A. (2011). Psychoseksuele ontwikkeling bij adolescenten met een autismespectrumstoornis: Ik Puber-training. Assen: Koninklijke Van Gorcum b.v.

Brown-Lavoie, S. M., Viecili, M. A., \& Weiss, J. A. (2014). Sexual knowledge and victimization in adults with autism spectrum disorders. Journal of Autism and Developmental Disorders, 44(9), 2185-2196.

Byers, E. S., Nichols, S., \& Voyer, S. D. (2013). Challenging stereotypes: Sexual functioning of single adults with high functioning autism spectrum disorder. Journal of Autism and Developmental Disorders, 43, 1-11.

Collins, R. L., Elliott, M. N., Berry, S. H., Kanouse, D. E., Kunkel, D., Hunter, S. B., et al. (2004). Watching sex on television predicts adolescent initiation of sexual behavior. Pediatrics, 114(3), e280-e289. 
Dewinter, J., Vermeiren, R., Vanwesenbeeck, I., \& van Nieuwenhuizen, C. (2013). Autism and normative sexual development: A narrative review. Journal of Clinical Nursing, doi:10.1111/jocn. 12397.

Gougeon, N. A. (2010). Sexuality and autism: A critical review of selected literature using a social-relational model of disability. American Journal of Sexuality Education, 5(4), 328-361.

Hellemans, H., Colson, K., Verbraeken, C., Vermeiren, R., \& Deboutte, D. (2007). Sexual behavior in high-functioning male adolescents and young adults with autism spectrum disorder. Journal of Autism and Developmental Disorders, 37(2), 260-269.

Hénault, I. (2006). Asperger's syndrome and sexuality: From adolescence through adulthood. London: Jessica Kingsley Publishers.

Hendriks, B., \& Meijs, P. (2005). Biobits VMBO: Voortplanting. Enschede: Printpartners Ipskamp.

Hudson, M., Nijboer, T. C. W., \& Jellema, T. (2012). Implicit social learning in relation to autistic-like traits. Journal of Autism and Developmental Disorders, 42(12), 2534-2545.

Hus, V., \& Lord, C. (2014). The autism diagnostic observation schedule, module 4: Revised algorithm and standardized severity scores. Journal of Autism and Developmental Disorders, 44(8), 1996-2012.

Kirby, D. B., Laris, B. A., \& Rolleri, L. A. (2007). Sex and HIV education programs: Their impact on sexual behaviors of young people throughout the world. Journal of Adolescent Health, 40(3), 206-217.

Kuo, M. H., Orsmond, G. I., Cohn, E. S., \& Coster, W. J. (2011). Friendship characteristics and activity patterns of adolescents with an autism spectrum disorder. Autism, 17, 481-500.

Lord, C., Risi, S., Lambrecht, L., Cook, E. H, Jr, Leventhal, B. L., DiLavore, P. C., et al. (2000). The autism diagnostic observation schedule-generic: A standard measure of social and communication deficits associated with the spectrum of autism. Journal of Autism and Developmental Disorders, 30(3), 205-223.

Maniglio, R. (2012). The Role of parent-child bonding, attachment, and interpersonal problems in the development of deviant sexual fantasies in sexual offenders. Trauma, Violence, \& Abuse, 13(2), 83-96.

Nichols, S., \& Blakeley-Smith, A. (2009). "I'm Not Sure We're Ready for This...": Working with families toward facilitating healthy sexuality for individuals with autism spectrum disorders. Social Work in Mental Health, 8(1), 72-91.

Orsmond, G. I., Krauss, M. W., \& Seltzer, M. M. (2004). Peer relationships and social and recreational activities among adolescents and adults with autism. Journal of Autism and Developmental Disorders, 34(3), 245-256.

Reitman, D., St Lawrence, J. S., Jefferson, K. W., Alleyne, E., Brasfield, T. L., \& Shirley, A. (1996). Predictors of African
American adolescents' condom use and HIV risk behavior. AIDS education and prevention, 8(6), 499-515.

Rock, E. M., Ireland, M., Resnick, M. D., \& McNeely, C. A. (2005). A rose by any other name? Objective knowledge, perceived knowledge, and adolescent male condom use. Pediatrics, 115(3), 667-672.

Ryan, S., Franzetta, K., \& Manlove, J. (2007). Knowledge, perceptions, and motivations for contraception influence on teens' contraceptive consistency. Youth \& Society, 39(2), 182-208.

Sevlever, M., Roth, M. E., \& Gillis, J. M. (2013). sexual abuse and offending in autism spectrum disorders. Sexuality and Disability, $31,1-12$.

Somers, C. L., \& Paulson, S. E. (2000). Students' perceptions of parent-adolescent closeness and communication about sexuality: Relations with sexual knowledge, attitudes, and behaviors. Journal of Adolescence, 23(5), 629-644.

Stokes, M., \& Kaur, A. (2005). High-functioning autism and sexuality: A parental perspective. Autism, 9(3), 266-289.

Stokes, M., Newton, N., \& Kaur, A. (2007). Stalking, and social and romantic functioning among adolescents and adults with autism spectrum disorder. Journal of Autism and Developmental Disorders, 37(10), 1969-1986.

Sullivan, A., \& Caterino, L. C. (2008). Addressing the sexuality and sex education of individuals with autism spectrum disorders. Education and Treatment of Children, 31(3), 381-394.

t HART-Kerkhoffs, L. A., Jansen, L. M., Doreleijers, T. A., Vermeiren, R., Minderaa, R. B., \& Hartman, C. A. (2009). Autism spectrum disorder symptoms in juvenile suspects of sex offenses. Journal of Clinical Psychiatry, 70(2), 266-272.

Tolman, D. L., \& McClelland, S. I. (2011). Normative sexuality development in adolescence: A decade in review, 2000-2009. Journal of Research on Adolescence, 21(1), 242-255.

Urbano, M. R., Hartmann, K., Deutsch, S. I., Polychronopoulos, G. M. B., \& Dorbin, V. (2013). Relationships, sexuality, and intimacy in autism spectrum disorders. In M. Fitzgerald (Ed.), Recent Advances in Autism Spectrum Disorders - Volume I. InTech. Available from: www.intechopen.com/books/recentadvances-in-autism-spectrum-disorders-volume-i/relationshipssexuality-and-intimacy-in-autism-spectrum-disorders.

Wechsler, D. (2004). The Wechsler intelligence scale for children (4th ed.). London: Pearson Assessment.

Wolters, C. A. (2003). Regulation of motivation: Evaluating an underemphasized aspect of self-regulated learning. Educational Psychologist, 38(4), 189-205.

Zimet, G. D., Bunch, D. L., Anglin, T. M., Lazebnik, R., Williams, P., \& Krowchuk, D. P. (1992). Relationship of AIDS-related attitudes to sexual behavior changes in adolescents. Journal of Adolescent Health, 13(6), 493-498. doi:10.1016/1054139X(92)90013-2. 